\section{$\underset{\substack{\text { hommes } \\ \text { \& migrations }}}{ }$}

\section{Hommes \& migrations}

Revue française de référence sur les dynamiques

migratoires

\section{$1296 \mid 2012$}

Le Mexique dans les migrations internationales

\title{
De la nécessité de lire Pascal au mois de janvier
}

\section{Mustapha Harzoune}

\section{(QpenEdition \\ Journals}

\section{Édition électronique}

URL : http://journals.openedition.org/hommesmigrations/1525

DOI : 10.4000/hommesmigrations. 1525

ISSN : 2262-3353

\section{Éditeur}

Musée national de l'histoire de l'immigration

\section{Édition imprimée}

Date de publication : 1 mars 2012

Pagination : 136-141

ISSN : 1142-852X

Référence électronique

Mustapha Harzoune, «De la nécessité de lire Pascal au mois de janvier», Hommes \& migrations [En ligne], 1296 | 2012, mis en ligne le 29 mai 2013, consulté le 22 septembre 2020. URL : http:// journals.openedition.org/hommesmigrations/1525; DOI : https://doi.org/10.4000/ hommesmigrations. 1525

Ce document a été généré automatiquement le 22 septembre 2020.

Tous droits réservés 


\title{
De la nécessité de lire Pascal au mois de janvier
}

\author{
Mustapha Harzoune
}

1 Le mois de janvier est le mois des étrennes et de la galette des rois. Des petits présents et de la petite fève. Celui aussi du bilan de la politique d'immigration. Janvier c'est Janus, le dieu romain des portes et des ouvertures. Dans le pays de Lévinas et de Derrida, l'hospitalité n'est plus le maître mot de ce bilan annuel. L'exercice consiste désormais à bomber le torse et à montrer ses biscotos, à plutôt exalter une politique de fermeture (maîtrise de l'immigration) et de porte claquée au nez des impudents (expulsions). L'argumentaire est connu : la crise, l'intégration... Alors exit les illégaux. Et les légaux! "Des lois Hortefeux et Besson aux démantèlements de campements illégaux en passant par le discours de Grenoble, le gouvernement n'aura cessé depuis 2007 de faire la chasse aux étrangers en situation irrégulière. Et régulière", dixit Libération du 24 janvier. Janvier mois de l'ouverture...

2 Et cette année, Libération du 11 janvier titre "Guéant frappe encore", quand Le Monde du même jour parle de "discours très dur". Il y avait trop d'immigrés clandestins; il faut maintenant réduire l'immigration légale à "150 000 entrées" par an. Plus que le FN, mais la logique est la même: allez ouste les basanés sinon gare à la rouste! Après l'immigration de travail (adieu l'immigration "choisie") et les étudiants (circulaire Guéant) sus aux demandeurs d'asile (57000 demandeurs cette année, en hausse de $8,2 \%$ ) et à la parentèle. Les expulsions continuent de grimper (en théorie) et l'objectif est de 35000 pour 2012.

3 Voici les grandes lignes de ce bilan selon le ministre: baisse du nombre de naturalisations (-30\%, de 94500 à 66000$)$; hausse des reconduites à la frontière (32 912) ; baisse des attributions des premiers titres de séjour de 3,6 \% (189 500 en 2010 et 182500 en 2011) ; baisse des titres de séjour délivrés à de nouveaux salariés étrangers de $26 \%$ (12 400 en 2010 et 9100 en 2011); baisse des titres de séjour pour "liens personnels et familiaux" de $14 \%$ (14600 en 2010 et 12600 en 2011). Ce bilan "ne manquera pas de donner lieu à une nouvelle controverse de chiffres" écrit Libération. 
D'ailleurs, pour Le Monde du jour, ces chiffres "restent à relativiser. Le record des reconduites à la frontière est en partie dû à l'éloignement des Roms" ; "la baisse de l'immigration de travail, elle, est sans doute moins due à la politique coercitive de M. Guéant qu'à la crise économique"; "l'indicateur de 'baisse de l'immigration familiale' [...] correspond, lui, seulement aux titres de séjour dits 'liens personnels et familiaux"'; “seuls les flux 'discrétionnaires' - soit ceux liés au travail - sont plus ou moins 'maîtrisables"'.

5 Tefy Andriamanana sur Marianne.fr du 11 janvier est sur le même registre : "il [Guéant ] oublie d'une part que la crise a aussi joué, au-delà des effets de sa propre politique. Avec la baisse de l'activité, on recrute moins, que ce soit des locaux ou des immigrés. Guéant oublie aussi qu'entre 2005 et 2010, l'immigration de travail a augmenté de près de 29,8 \%." Côté expulsions, "au premier semestre 2011, selon la députée UMP Béatrice Pavy, seules 29,5\% des décisions d'expulsion ont débouché sur une réelle sortie du territoire contre 32,2\% au 1er semestre 2010." À cela, il ajoute "la piste roumaine, l'autre dada de Claude Guéant. Pour les expulser et gonfler ses chiffres, le ministre compte sur l'aide au retour humanitaire [...]. Le gouvernement est accusé de forcer un peu trop la main à certaines populations défavorisées notamment les Roms pour qu'ils acceptent ce chèque... quitte à revenir en France ensuite." Conclusion de l'auteur : "La politique du chiffre de Guéant et Sarkozy n'est donc qu'une politique d'affichage obtenue par quelques subtils bricolages et une bonne dose de propagande."

6 Le 11 janvier La Cimade note aussi "que $30 \%$ environ des expulsions correspondent à des retours 'volontaires forcés' de citoyens européens, roumains ou bulgares qui ont tout à fait le droit de revenir en France" et que "près d'un autre tiers des expulsions correspond à des réadmissions vers d'autres pays européens de personnes interpellées aux frontières terrestres de la France." Elle dénonce les effets pervers des réductions du nombre de titres de séjour : "C'est seulement condamner à la précarité et à la clandestinité des milliers d'hommes, de femmes et de familles qui vivent et travaillent en France sans aucun effet sur une prétendue pression migratoire dont serait victime la France."

7 Le 21 janvier, Libération remet le couvert sur l'immigration de travail : “Claude Guéant n'a retenu qu'une des catégories de l'immigration professionnelle, les premiers titres de séjour salariés. Il a exclu de ses statistiques les saisonniers, les actifs non salariés, les travailleurs temporaires, les régularisés au titre du travail, ainsi que les étudiants étrangers qui ont pris le statut de salarié à la suite de leurs études [...]." Selon les statistiques de l'office français de l'immigration et de l'intégration, basées sur les visites médicales obligatoires des migrants, "l'immigration professionnelle, tout compris, a progressé de $5 \%$ par rapport à 2010".

8 Au temps joyeux de l'immigration "choisie", "l'amusant, écrit Libé, est que Brice Hortefeux s'était livré [...] ’à la même opération que Guéant... mais dans l'autre sens". "Ce qui avait permis à Hortefeux de se féliciter en février 2009 d'avoir accordé 33000 titres de séjour au titre de l'immigration de travail. Trois ans après, Guéant donne le chiffre de 9154 . Cela ne veut pas dire que le flux a baissé de 24000 en trois ans. Cela veut simplement dire que la méthode de calcul retenue varie selon les objectifs assignés. Une règle d'or de la statistique à usage gouvernemental."

\section{Délinquance et immigration}

Le 15 février, l'Observatoire national de la délinquance et de la réponse pénale (ONDRP) rendait public son rapport sur les "Français et étrangers mis en cause par la police et la gendarmerie pour atteintes aux biens ou pour atteintes volontaires à l'intégrité physique" entre 
2006 et 2011. Sur les 305708 personnes interpellées pour un vol ou pour un délit de destruction ou de dégradation, 52751 (17\%) seraient de nationalité étrangère. La part des étrangers dans les atteintes aux personnes s'élèverait à $12 \%$. Pour le site marocain Yabiladi.com (16 février), "ce qui est le plus alarmant, ce ne sont pas les chiffres détaillés dans le rapport mais plutôt le fait que les étrangers qui commettent ces crimes soient classés par nationalité." "Ainsi on apprend que les Roumains sont les premiers à être mis en cause par cette étude. Les actes qu'ils ont commis ont progressé de plus de $114 \%$ entre 2008 à 2010". Viennent ensuite les Algériens (+ $30 \%$ ) et les Marocains (+ $21 \%)$ : “3 032 Marocains ont été mis en cause par la police ou la gendarmerie pour vol en 2008, 3469 en 2009 et 3676 en 2010."

Dès le 18 janvier, le ministre de l'Intérieur enfourchait son cheval de bataille : le lien entre immigration et délinquance. "Pour M. Guéant, la délinquance commise par les étrangers est 'supérieure à la moyenne' et elle appelle des 'mesures spécifiques' à prendre 'dès les prochains mois"' (Le Monde). Déjà, "le ministre lance le 'buzz"' autour de l'étude (à paraître) de l'ONDRP dont la "nouveauté" souligne aussi Le Monde est "la répartition par nationalité". L'étude "affirme que le nombre d'étrangers mis en cause pour cambriolage a augmenté de $40 \%$ depuis 2008". "Le 10 janvier, écrit Le Monde, sur RMC Info, M.Guéant estime que la hausse prévisible du nombre de cambriolages en 2011 'est très liée à la délinquance étrangère'." La veille, il déclarait: "Les cambriolages de résidences principales et secondaires sont en augmentation de $7 \%$, c'est dû à un phénomène nouveau qu'il est très difficile de combattre, qui est celui des 'raids' menés par des personnes originaires d'Europe centrale et orientale."

11 Sur Marianne.fr, Tefy Andriamanana s'est penché le 9 janvier sur le bilan annuel du ministère de la Justice. "En 2010, 12,64 \% des condamnés pour crimes (punissables de plus de 10 ans de prison) étaient des étrangers, cette proportion est de 12,58\% pour les délits. [...] Quand on observe les évolutions entre 2007 et 2010, la part de condamnés étrangers varie entre 12,23\% et 12,64\% pour les crimes et entre 11,8\% et 12,58\% pour les délits. [...] En deux ans [2008/2010], la part des étrangers condamnés a augmenté de 3,35\% pour les crimes et de 6,6\% pour les délits."

"Il y a donc une augmentation de la délinquance et de la criminalité des étrangers. Mais au vu des proportions mesurées et de la durée évoquée (deux ans), elle incite à reconsidérer sérieusement les chiffres venus de la police qui fuitent à ce sujet. En septembre dernier, la Préfecture de police de Paris évoquait, pour les sept premiers mois de 2011, une hausse de $90 \%$ en un an des mis en cause (et non des condamnés) roumains dans Paris et sa banlieue."

Le 5 janvier, sur son blog, le chercheur Laurent Mucchielli détaillait les quelque 630000 condamnations prononcées en 2010. Elles sanctionnaient 940000 infractions dont 93,4\% relevant de délits: infractions routières (35\%), "infractions en matière de stupéfiants" (15\%) dont $70 \%$ pour usage ou détention. "L'ensemble des vols et recels n'arrivent qu'en troisième position avec quelque 127000 infractions sanctionnées. Puis ce sont les violences physiques avec un peu moins de 80000 infractions sanctionnées (dont $80 \%$ parmi les moins graves selon le critère du nombre de jours d'ITT - incapacité totale de travail). “

Sur ces 630000 personnes condamnées, "82,2\% des condamnés étaient de nationalité française, $12,7 \%$ de nationalité étrangère. Les nationalités les plus fréquentes parmi ces personnes condamnées étaient à égalité les Algériens et les Marocains, devant les Portugais, les Roumains, les Tunisiens et les Turcs". 


\section{Délinquance, discriminations et méthode}

15 Le même Laurent Mucchielli rappelant sur son blog le 11 janvier les "fausses évidences statistiques [et les] vraies manipulations politiques", évoque “les multiples discriminations' qui, depuis les contrôles au faciès jusqu'à l'exécution des peines, expliquent au moins en partie la 'surreprésentation' des personnes de nationalité étrangère dans la délinquance enregistrée par les institutions pénales."

16 Ainsi, "le fait d'avoir la peau noire entraîne un risque d'être contrôlé 3 à 11 fois supérieur selon les sites, et le fait d'avoir le type maghrébin un risque 2 à 15 fois supérieur selon les sites étudiés ". Cette discrimination au faciès altère le sérieux de la méthode : "Les personnes mises en cause par la police [ne] constituent [pas] un échantillon représentatif de la délinquance constatée, a fortiori de la délinquance réelle" sans oublier "le fait que certaines infractions concernent par définition les étrangers" (infractions à la police des étrangers, faux documents d'identité et autres documents administratifs, délit de travail clandestin). “ Pour calculer un taux d'étrangers parmi les mises en cause policières, il faut donc les soustraire."

De plus, "les étrangers mis en cause ne sont pas nécessairement des personnes résidant sur le territoire national" mais des personnes transitant en France, comme "certaines délinquances (trafic de drogue, proxénétisme...) sont par définition transfrontalières et certains ressortissants étrangers peuvent donc être arrêtés pour des délits commis en France lors même qu'ils n'y résident pas".

18 Enfin, sur le plan judiciaire, "les étrangers sont plus lourdement sanctionnés que les nationaux, notamment plus souvent condamnés à des peines de prison, avec moins de sursis et des peines plus longues".

19 Sur ce sujet, après les études de l'Open Society Justice Initiative (2007) et du CNRS (2008) "qui concluait que les probabilités de contrôle sont 'six fois plus élevées pour les Noirs et près de huit fois plus pour les Arabes"', "dans un rapport, publié jeudi 26 janvier, l'ONG Human Rights Watch (HRW) dresse un constat sans concession des contrôles d'identité par les forces de police en France" (Le Monde, 27 janvier). "Ces conclusions ont été vivement contestées" par la Direction générale de la police nationale, les syndicats Alliance et Synergie. "Depuis 2006, 26 policiers sur les 145000 en exercice ont été sanctionnés pour comportement raciste."

Dans Le Monde du 1er février, les sociologues Philippe Robert et Renée Zauberman, offrent aux lecteurs un petit bijou de pédagogie citoyenne quant au bon usage de la statistique. Ils rappellent trois règles essentielles : "aucun chiffre, s'il reste isolé, ne mesure la délinquance"; "seule l'observation sur le long terme permet de comprendre la tendance de la délinquance"; enfin, "la mesure de la délinquance est une activité scientifique : elle doit être mise à l'abri des approximations politiques, administratives, voire corporatives. Or, qu'entend-on dans la présentation de la délinquance en 2011 faite par le ministre de l'Intérieur? D'abord, la flambée du cambriolage est attribuée à des 'raids d'étrangers'. Comment le sait-on? À peu près $88 \%$ des cambriolages (de résidence principale ou secondaire) ne sont jamais élucidés; autrement dit, la police n'identifie les cambrioleurs que dans un cas sur dix..." "La mesure de la délinquance ne deviendra crédible que si un panel de (vrais) scientifiques, spécialistes [...] reconnus par leurs pairs, est chargé de dresser un état de la délinquance [...]. Chacun sent bien, confusément, que les politiques de sécurité sont plus ou moins dans un cul-de-sac. L'incapacité des organismes publics à établir un diagnostic lucide sur la criminalité n'y est-elle pas pour beaucoup?" 


\section{Pascal plutôt que Descartes}

21 Sur le site du Point, le 16 février, Edgar Morin explique pourquoi il préfère Pascal à Descartes: "Parce qu'il a rompu avec la simplification et la causalité linéaire en une phrase géniale et définitive: 'Toutes choses étant causées et causantes, aidées et aidantes, médiates et immédiates [...], je tiens impossible de connaitre le tout sans connaître les parties non plus que de connaitre le tout sans connaitre particulièrement les parties". Il ajoute: "Descartes est un grand philosophe, mais sa méthode est trop analytique: il sépare les problèmes en petits morceaux qu'il traite ensuite séparément. Le contraire de Pascal. C'est en ce sens qu'il faut, à mon sens, sortir du cartésianisme."

Une analyse pascalienne de l'immigration et non le saucissonnage politico-électoral reviendrait peut-être sur ce que Le Monde du 18 janvier rapporte : "En France, la vitalité démographique va diminuer selon l'Insee." "Les générations du baby-boom vieillissant, le nombre de décès augmentera, même si l'espérance de vie continue à progresser. Si le nombre de naissances n'augmente pas, le solde naturel devrait donc diminuer et la croissance démographique ralentir, de façon progressive, pendant les trente prochaines années."

Pascal et sa méthode ne mépriseraient pas non plus l'étude des besoins sectoriels de l'économie française. Ainsi, dans Le Monde du 22 février, Daniel Bloch déplore que "la France manque d'ingénieurs et de scientifiques pour innover et rendre son économie et son industrie plus compétitives". Comment répondre à ce défi? L'auteur propose de reconsidérer les cursus des filières technologiques. Ne faut-il pas aussi examiner la politique d'immigration sous cet angle ? Comme dans le secteur de la santé.

Pour permettre à des milliers de médecins étrangers de continuer à exercer, "le 24 janvier, les sénateurs ont ratifié à l'unanimité une proposition de loi du député UMP Jean-Pierre Door prolongeant du 31 décembre 2014 au 31 décembre 2016 la date butoir pour valider une épreuve de vérification des connaissances et donc pour pouvoir continuer à exercer". 4000 praticiens, médecins, pharmaciens, chirurgiens-dentistes et sages-femmes étrangers seraient concernés par cette autorisation d'exercer. "Venus souvent d'Afrique noire francophone ou du Maghreb, ces praticiens pallient depuis de nombreuses années les difficultés de recrutement des hôpitaux français, où ils représentent parfois jusqu'à $30 \%$ du personnel" (Le Monde, 26 janvier).

Dans ce domaine encore, la complexité pascalienne éviterait de fabriquer des boucs émissaires (voir l'adresse du docteur Madjid Si Hocine invitant "Monsieur Guéant" à ne pas se "mêler du trou de la Sécurité sociale" dans Libération du 2 janvier) et d'entonner le refrain sur l'attrait des prestations sociales sans se demander pourquoi les immigrés affluent aussi nombreux vers l'Allemagne, qui, rappelle Bertrand Nouel (Le Monde, 17 février) "a réduit considérablement l'indemnisation du chômage (12 mois pour les moins de 50 ans)" et où "les chômeurs sont contraints d'accepter tout emploi proposé, même peu rémunérateur".

26 Mais voilà, "le vertige de l'évaluation à tous crins" gâte aussi les esprits. "Nous sommes passés en quelques années du souci de valorisation à l'impératif d'évaluation", écrit Michel Chauvière dans L'Intelligence sociale en danger. Chemins de résistance et propositions (La Découverte, 2011). "L'évaluation s'impose désormais comme unique morale publique, ironise M. Chauvière, en dénonçant une 'approche mécanique du social"' (Le Monde, 10 janvier). Ainsi cherche-t-on à évaluer jusqu'à la nausée "le rapport 'coût-bénéfice' des migrations". Pourtant, "tous les économistes sérieux qui ont travaillé la problématique sont plutôt arrivés à 
la conclusion que l'immigration avait soit un impact 'légèrement positif' sur le budget des États, soit "légèrement négatif" (Le Monde, 14 janvier). Et de montrer qu'une pensée autrement complexe n'ignore pas que les politiques d'immigration procèdent aussi des cadres juridiques, constitutionnels, des accords de partenariat et autres conventions internationales... Encore Pascal.

Quid enfin des émigrés français, ces expatriés (c'est plus chic qu'immigré, non ?) qui selon Pierre-Yves Geoffard, professeur à l'École d'économie de Paris, directeur d'études à l'Ehess, contribuent plus sûrement que "nos" immigrés renvoyés à leur religion à l'éclatement, "par le haut", de la nation (Libération, 21 février) ? "Curieusement, les départs de Français qui vont s'installer à l'étranger ne semblent pas concerner les débats sur la nation. $E t$, lorsqu'il s'agit de l'exil de nos riches compatriotes, la question devient économique; il n'est plus question de morale; il faut tout faire pour qu'ils restent en France, parce que leurs talents, leurs compétences, bénéficient à tous, forcément." Et de citer Serge Dassault : "L'ISF est une catastrophe qui a fait fuir des milliers d'industriels, beaucoup sont partis et les autres vont partir car ils ont peur des socialistes. Ceux qui vont créer de la richesse, faire de la croissance, ce n'est pas les pauvres. C'est les riches. Il faut garder les riches." Alors Pierre-Yves Geoffard convoque Renan, les "sacrifices", "le désir" de continuer "la vie commune" et le "plébiscite de tous les jours". Conclusion: "Ces riches qui menacent de partir: ce sont eux qui sapent la nation. Qui peut croire que la nation, aujourd'hui, est menacée par l'immigration? Comment, au contraire, ne pas voir qu'en s'acharnant sur les étrangers, qu'ils soient jeunes qualifiés ou pauvres malades, ces politiques ne font que détourner l'attention des vrais enjeux : la nation est avant tout fragilisée par les comportements des Français les plus riches. Que ceux-ci cherchent à défendre leurs intérêts, après tout, c'est assez naturel; que les politiques les encouragent est une autre affaire!" 